\title{
Preparation of experiments to study light nuclei structure at Nuclotron
}

\author{
A.A Terekhin*, S.M. Piyadin, V.P. Ladygin, A.N. Khrenov, A.Yu. Isupov, Yu.V. Gurchin, \\ V.A. Krasnov, A.K. Kurilkin, P.K. Kurilkin, S.G. Reznikov and T.A. Vasiliev. \\ Joint Institute for Nuclear Researches, Joliot-Curie St., 6, Dubna, 141980, Russia \\ E-mail:aterekhin@jinr.ru

\begin{abstract}
The status of preparation of the BM@N- and DSS-experiments at Nuclotron-JINR is reported. The results of the first extraction of the $3.42 \mathrm{~A} \cdot \mathrm{GeV}$ carbon and 4.0 A.GeV deuteron beam at Nuclotron and their transportation to the beam experimental area are presented. The measurements of dp-elastic scattering, dW- and dAg- quasi-elastic scattering at internal target at Nuclotron are performed.
\end{abstract}

XXI International Baldin Seminar on High Energy Physics Problems

Dubna, Russia

September,10-15 2012

${ }^{*}$ Speaker. 


\section{Contents}

$\begin{array}{ll}\text { 1. Introduction } & 2\end{array}$

2. Experimental setup for the BM@N experiment 2

3. Results of measurements at extracted beam 3

4. Measurements at internal target $\quad 4$

5. Conclusion 6

\section{Introduction}

The study of the dense baryonic matter at Nuclotron (BM@N project) [1] is one of the main scientific directions at the LHEP-JINR. The research program of BM@N project will be focused on the production of strange matter in heavy-ion collisions at beam energies between 2 and 6 A. GeV. For these purposes it is proposed to install an experimental setup at the $6 \mathrm{~V}$ beamline in the fixed-target hall at Nuclotron.

The goal of DSS project [2] is the systematic study of the polarization observables in hadronic reactions by using polarized deuteron beam and polarized ${ }^{3} \mathrm{He}$ - target [3] at intermediate and high energies. The experiment to measure $T_{20}$ and $C_{y y}$ for the ${ }^{3} \mathrm{He}(d, p){ }^{4} \mathrm{He}$ - reaction at 1.0-2.0 GeV deuterons energy is planned. The results of this experiment will help to understand the short-range deuteron spin structure.In addition the study of deuterons interaction with protons and nuclei is included in DSS project.

The results of preparation of the experiments at the extracted beam and internal target station at Nuclotron-JINR are reported. First extraction of the $3.42 \mathrm{~A} \cdot \mathrm{GeV}$ carbon beam and of the 4 A. GeV deuteron beam [4] at Nuclotron and their transportation to the beam experimental area and the measurements of dp-elastic scattering, $\mathrm{dW}$ - and dAg- quasi-elastic scattering at internal target station at Nuclotron are performed.

\section{Experimental setup for the BM@N experiment}

The experimental setup of the experiment the extracted beam at Nuclotron is placed at the distance of about $110 \mathrm{~m}$ from the ring of Nuclotron. The magnetic scheme of the $6 \mathrm{~V}$ line is shown in Fig. 1. The doublet of quadrupole magnets (QL) allows to focus the beam. Two small dipole magnets M1 and M2 can deflect the beam in the horizontal and vertical directions. The large aperture magnet SP41 (M3) can be used as an analyzing magnet. The start scintillation counter S1 is based on the use of XP2020 PMT. The stop scintillation counter S2 is based on the use of PMT 


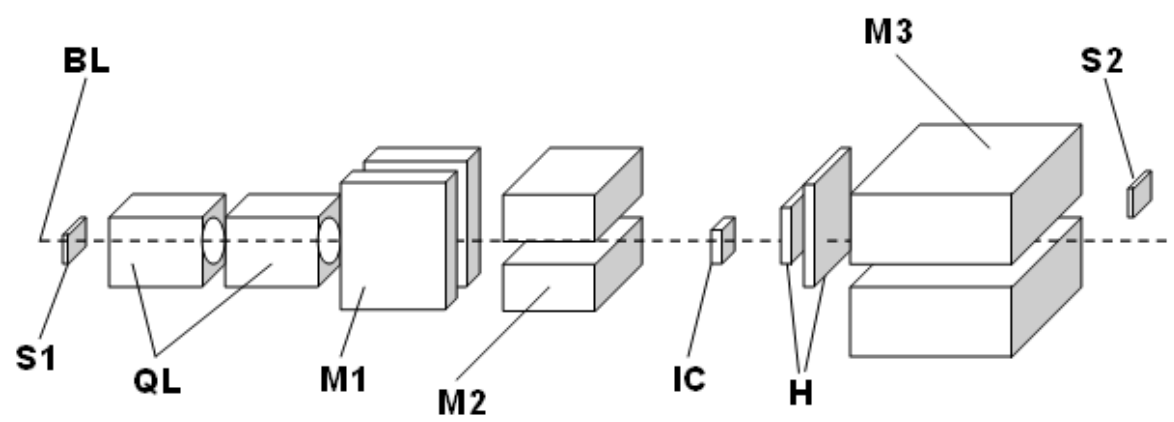

Figure 1: The layout of the experiment. BL is the optical axis of the 6V beam line, QL and M1-M3 are the quadrupole and dipole magnets, respectively. IC is the coordinate ionization chamber, $\mathbf{S 1}$ and $\mathbf{S 2}$ are the start- and stop- scintillation counters, respectively, $\mathbf{H}$ are the 2 planes of the scintillation counters hodoscopes.

FEU-87. The additional multiwire ionization chamber (IC) and two scintillation hodoscopes (H) are installed in the experimental area.

The first and second hodoscopes provided the coordinate measurements in the horizontal and vertical planes, respectively. Each hodoscope (see Fig. 2) consist of 8 scintillators with the size of 400x40x4 $\mathrm{mm}^{3}$ viewed by PMTs FEU85 from both sides each [5].

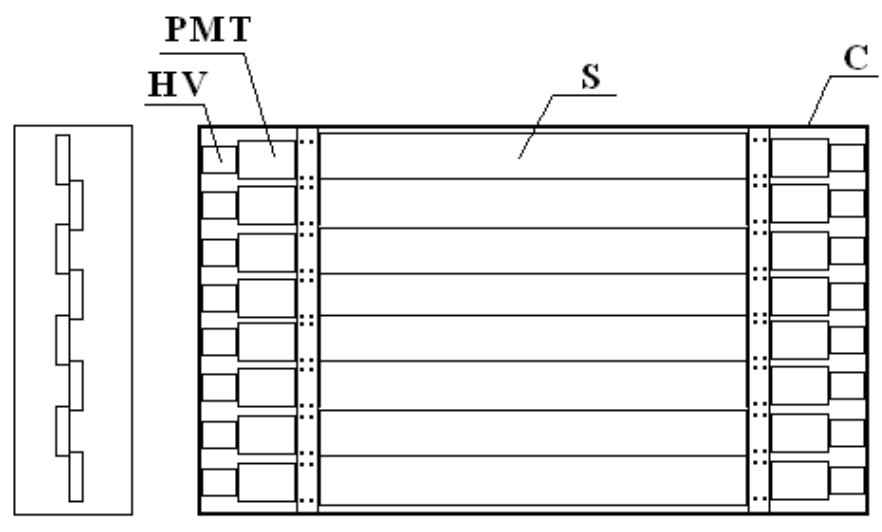

Figure 2: Left: profile of the hodoscope. The scheme of the hodoscope. Right: the layout of the hodoscope. C - case, HV - cells of high-voltage power,PMT - photomultipliers FEU-85, S - scintillation plates

The VME based data acquisition system was used for the data taking from scintillation detectors. 16 - channel time and charge digitizer (TQDC16) allows to measure the amplitude and time appearance of the signal [6].

\section{Results of measurements at extracted beam}

The carbon- and deuteron- beams with energies 3.42 and $4 \mathrm{~A} \cdot \mathrm{GeV}$, respectively, were successfully transported to the $6 \mathrm{~V}$ - area. The correlation of the signal amplitudes taken from the both sides of one of the scintillation detector of hodoscope for carbon nuclei beam is shown in Fig. 3. One can see the clean separation of the primary carbon nuclei and background single charged particles. The X-Y coordinates correlation for carbon nuclei was plotted (see Fig. 4). One can see that the 
size of the carbon beam in Y- direction is wider. The amplitude distribution for the deuteron beam (see Fig. 5) corresponds to amplitude distribution for the single charged particles. The inclusion of quadrupole magnetic lenses allowed to reduce the size of the beam (see. Fig 6).

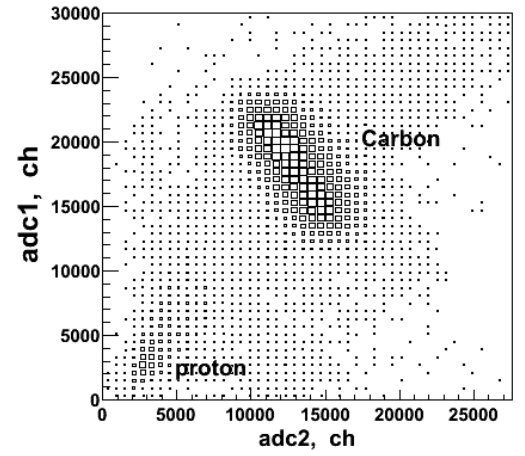

Figure 3: The signal amplitudes correlation for carbon nuclei beam for one of the scintillation detector of the hodoscope.

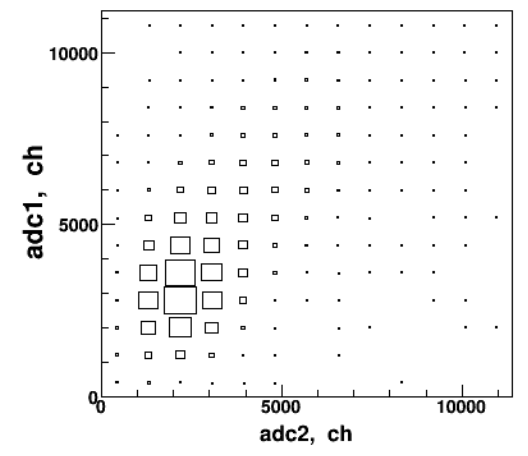

Figure 5: The signal amplitudes correlation for deuteron beam for one of the scintillation detector of the hodoscope.

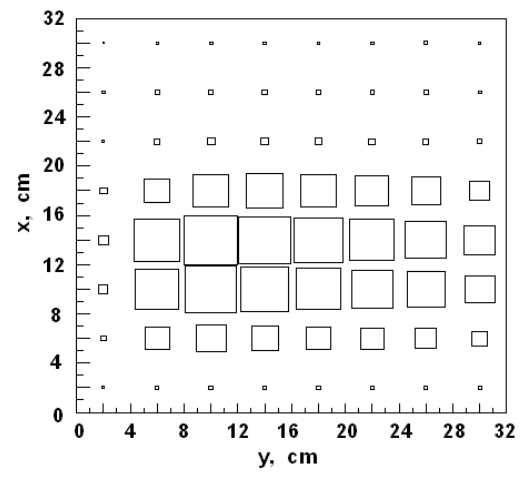

Figure 4: The X-Y coordinates correlation for carbon nuclei.

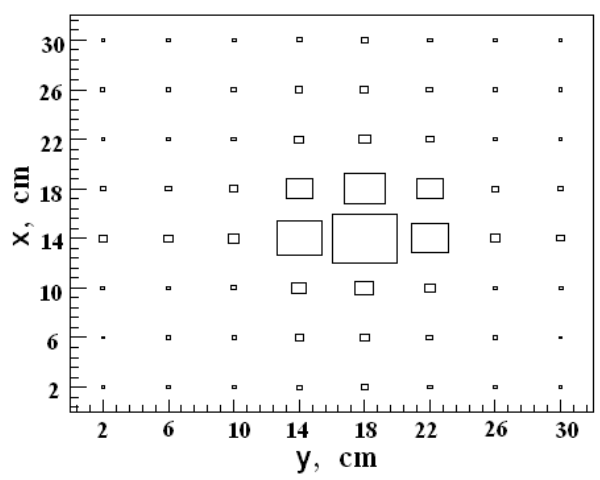

Figure 6: The X-Y coordinates correlation for deuteron beam.

\section{Measurements at internal target}

In addition the methodical measurements to obtain of the differential cross section for dpelastic scattering at the ITS was performed. The measurements have been performed by using $\mathrm{CH}_{2}$-target and deuteron beam at $1 \mathrm{GeV} /$ nucleon energy. The dp- and pp-detectors were mounted at angle $\theta^{*}=75^{\circ}$ and $\theta^{*}=90^{\circ}$ in the c.m.s., respectively. Subtraction of the time signal taken from the D- and P- detectors for counters based on the FEU -85 and counters based on the Hamamatsu H7416MOD is shown in Fig.7 and Fig. 8 respectively. One can see, that the dp-elastic scattering events and background are not selected in the case of the measurements with the counters based on the FEU -85 unlike the counters based on the Hamamatsu H7416MOD. 


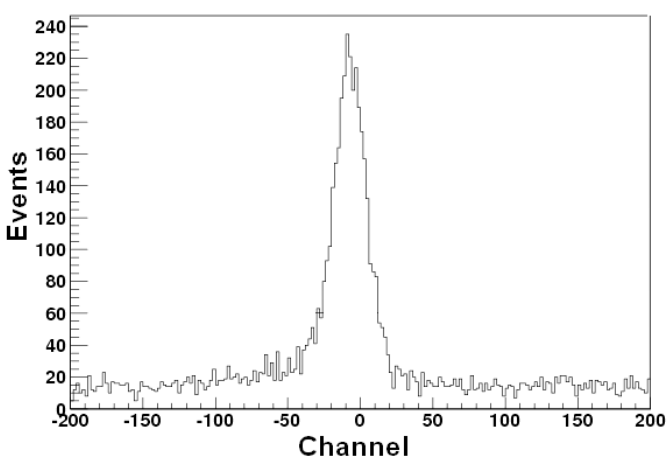

Figure 7: Subtraction of the time signal from D- and Pcounters. The data were obtained by using the counters based on the FEU-85.

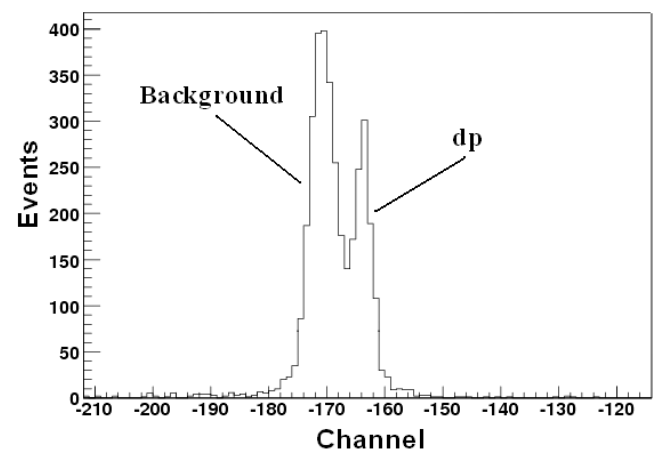

Figure 8: The subtraction of the time signal from $D-$ and P- counters. The data were obtained by using the counters based on the Hamamatsu H7416MOD.

The anomalous pion yield is observed in the interaction of protons and deuterons with nuclei [7]. The experiment at ITS with DELTON setup [8] on the measurement of the pion yield in the interact of deuterons with W and Ag nuclei was performed in 2011 - 2012 years. During these experiments the energy dependence of the dp- and pp-yield in $\mathrm{dW}$ and $\mathrm{dAg}$ - interactions have been measured. The reaction products were registered by the scintillation counters. Each counter consist of two detectors with thin and thick scintillators [9]. First particle passes in a thin plastic scintillator and then in thick plastic scintillator. The thin scintillator is used to reduce the background. The set of four detectors to register the reaction products is placed in horizontal plane. D- and P- counters are to register dp-elastic scattering. PPL and PPR - to register pp- quasi-elastic scattering. The monitor-counters are mounted in the vertical plane. The results of these measurements are shown in Fig. 9 and Fig 10, respectively. Any anomalous effect isn't observed for studied channels.

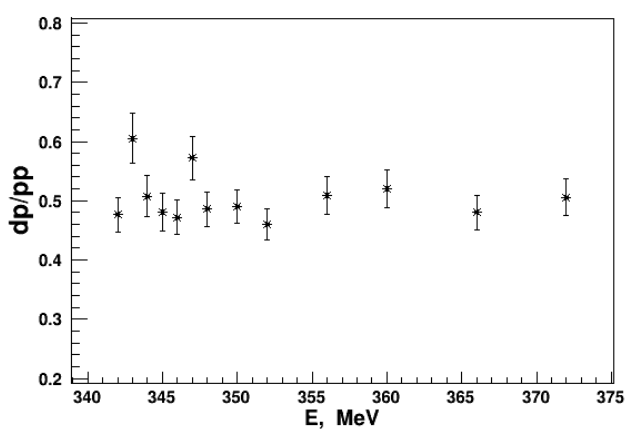

Figure 9: Energy dependences of the dp- to pp-quasielastic yields ratio for $\mathrm{dW}$-interaction.

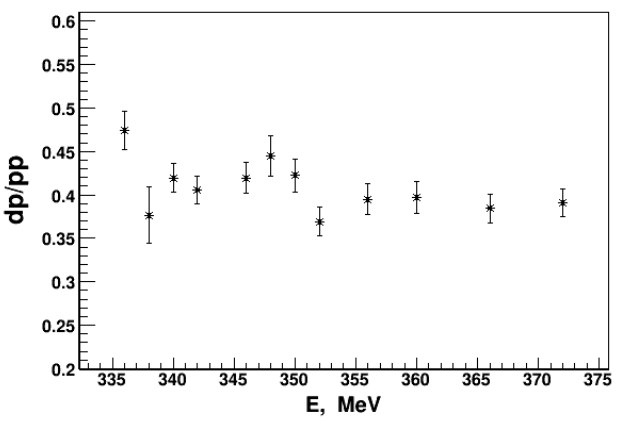

Figure 10: Energy dependences of the dp- to pp-quasielastic yields ratio for $\mathrm{dAg}$-interaction.

Measurements with the omission of target cycles was performed in regime of the simultaneous run at ITS and at the extracted beam setup. The target was injected out from the beam every third, every second and every fourth cycle. One can see in Fig. 11 the statistic of the extracted beam setup is increased when the target doesn't cross beam, unlike the statistic of ITS setup. 


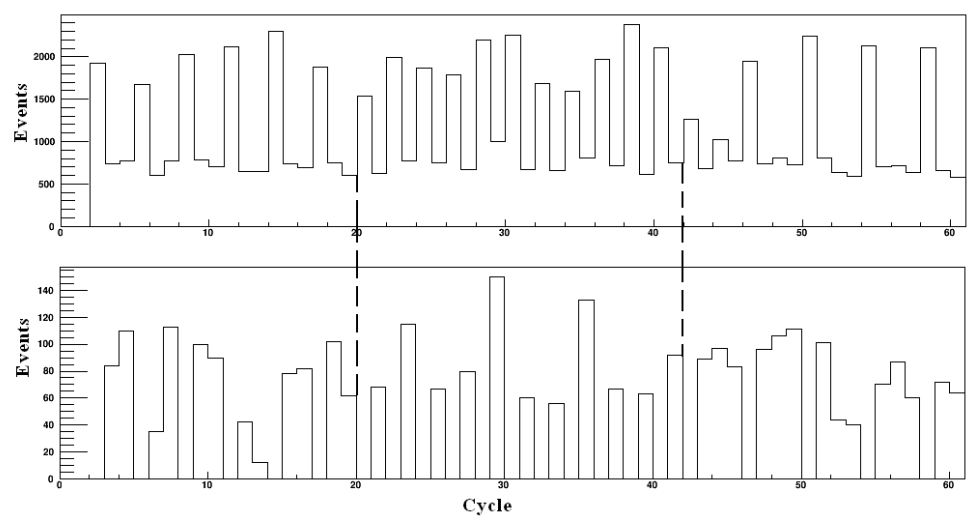

Figure 11: Distribution of events over cycles recording. Upper - for extracted beam setup, bottom - for ITS

\section{Conclusion}

First extraction of the $3.42 \mathrm{~A} \cdot \mathrm{GeV}$ carbon beam and $4 \mathrm{~A} \cdot \mathrm{GeV}$ deuteron beam at Nuclotron and their transportation to the experimental area are performed. Work of magnetic optic elements are demonstrated.

Measurements of dp-elastic scattering, dW- and dAg- quasi-elastic scattering at internal target station at Nuclotron are performed. Energy dependences of dp- and pp-quasi-elastic scattering was measured. Any anomalous effect isn't observed for these channels.

The regime of the beam sharing between the experiment at ITS and extracted beam is realized. The simultaneous work of the ITS and extracted beam setup is demonstrated.

We thanks J.Lukstins and A.N. Livanov for their help during preparation of the experiment. The work has been supported in part by the RFBR grant 10-02-00087a.

\section{References}

[1] http://nica.jinr.ru

[2] V.P. Ladygin et al. Short-range correlation studies in collisions of polarized nuclei at Nuclotron-M, EPJ Web of Conferences 2010. 3. 04004.

[3] T. Uesaka et al. Nucl.Instr.Meth. in Phys.Res. A402 (212) 1998.

[4] S.M. Piyadin et al. First extraction of the $3.42 \mathrm{AGeV} \mathrm{C} \mathrm{beam} \mathrm{for} \mathrm{studies} \mathrm{of} \mathrm{baryonic} \mathrm{matter} \mathrm{at}$ Nuclotron, JINR Preprint E1-2012-17 2012.

[5] A.A. Terekhin et al. Scintillation hodoscope for experiments at extracted beam at Nuclotron JINR, BSU Scientific bulletin, MathematicsPhysics 11(130) (207) 2012.

[6] http://afi.jinr.ru/TQDC-16

[7] V.A. Krasnov et al. Search for resonant structure in the pion production reaction on the Nuclotron internal beam, Int.J.Mod.Phys. A22 (604) 2007

[8] V.A. Krasnov et al. Multilayer Scintillation Charge Pions Spectrometer, JINR Preprint P13-2011-135 2011.

[9] Yu.V. Gurchin et al. Study of the possibility to use d p-elastic scattering for the nuclotron external deuteron beam polarimetry, Phys.Part.Nucl.Lett. 8 (566) 2011. 